J. Clin. Chem. Clin. Biochem.

Vol. 18, 1980, pp. 275-278

\title{
Kinetische Glucosebestimmung nach der Glucosedehydrogenase-Methode mit dem Analysenautomaten ACP 5040 (Eppendorf)
}

\author{
Von A. Bruckner \\ Zentrallaboratorium (Chefarzt Dr:med. W. Fritzsche) des Städtischen Krankenhauses Frankfurt/M.-Höchst
}

(Eingegangen am 10. Oktober 1979/2. Januar 1980)

Zusammenfassung: Es wird eine reaktionskinetische Glucosebestimmung in Blut, Urin und Liquor mit Hilfe des neuen Eppendorf Analysenautomaten ACP 5040 beschrieben. Dabei entsteht aus Glucose mittels Glucosedehydrogenase Gluconsäure, $\mathrm{NAD}^{+}$wird zu NADH reduziert. Die von uns ausgearbeitete Bestimmungsvariante zeichnet sich durch gute Präzision, Richtigkeit und Durchsatzgeschwindigkeit aus. Die Korrelation mit der Hexokinase/Glucose6-phosphatdehydrogenase-Endpunk tmethode ist gut.

\section{The kinetic determination of glucose with the glucose dehydrogenase method using the Eppendorf automatic} analyzer 5040

Summary: A kinetic determination of glucose from blood, urine and spinal fluid is described with use of the new automatic analyzer ACP 5040 Eppendorf. The method uses glucose-dehydrogenase which converts glucose to gluconic acid. The NADH formed can be measured by the increase in absorbance at $334 \mathrm{~nm}$. Our variation of test methodology gives good precision, accuracy and a high performance speed. There is a good correlation with the hexokinase-glucose-6-phosphate-dehydrogenase end point method.

\section{Einführung}

Die Glucosebestimmung ist nach wie vor eine der häufigsten Bestimmungen im klinisch-chemischen Laboratorium. Wir beschreiben in dieser Arbeit eine, unseres Wissens erste, Methodenadaptation an den ACP 5040. Die Methode zeichnet sich durch Wirtschaftlichkeit und hohe Präzision aus. Um einen besseren Vergleich mit der Hexokinase-Methode zu erzielen, haben wir die Untersuchungen aus Perchlorsäure:Uberstand durchgeführt. Die für die Bestimmung verwendete Glucosedehydrogenase (EC 1.1.1.47) erfüllt chemische Prinzipien für die kinetische Substratbestimmung im ,pseudo-kinetischen" Vèrfahrèn. Das Enżym besitżt eiñe sehr lange Stabilität, auch bei Raumtemperatur. Thiolgruppenoxidierende Stoffe können das Enzym nicht hemmen, da es keine SH-Gruppen besitzt; Schwermetalle und Chelatbildner inihibieren es ebenfalls nicht (1). Das Enzym besitzt einen $K_{\mathrm{m}}$-Wert für Glucose von $10^{-2}$ $\mathrm{mol} / 1$ (pH 8) und einen $K_{\mathrm{m}}$-Wert für $\mathrm{NAD}^{+}$von $1,6 \times 10^{-3} \mathrm{~mol} / 1$ (pH 8). Damit ist die theoretische Voraussetzung, daß $[\mathrm{S}] \ll K_{\mathrm{m}}$ ist, auch bei einer Glucose- konzentration von $55,5 \mathrm{mmol} / \mathrm{l}$ noch gegeben. So ist die Gleichung

$$
\mathrm{v}=-\frac{\mathrm{d}[\mathrm{S}]}{\mathrm{dt}}=\frac{\mathrm{V}}{K_{\mathrm{m}}} \times[\mathrm{S}]=\mathrm{k}^{\prime} \times[\mathrm{S}]
$$

bei unten beschriebenen Probe-Reagenz-Verhältnissen erfüllt, da der Wert von [S]/Km kleiner als 0,05 ist; d.h. $v$ ist mit $[S]$ linear proportional $(2,3)$.

\section{Material und Methoden}

\section{Reagenzien}

1. Pufferlösung Art. Nr. 14051, Fläschchen Enzymgemisch (enthält Glucosedehydrogenase und Mutarotase), Fläschchen Coenzym $\left(\mathrm{NAD}^{+}\right)$, beide aus System Glucose, Glucosedehydrogenase-Methode, UV-Test Art. Nr. 14055, GlucoseStandardlösung von 2,78 bis $27,75 \mathrm{mmol} / 1$ Art. Nr. 9423/1-5, $D(+)$ Ǵlucose wasserfrei, für biochemische $Z$ wecke Art. Nr. 8337, Benzoesäure p.a. Art. Nr. 136 von E. Merck AG (Darmstadt).

2. Perchlorsäure etwa $0,33 \mathrm{~mol} / 1$ Art. Nr. 125369 , Test-Combination, Glucose, Hexokinase-Methode, UV-Test Art. Nr. 
124346, Kontrollseren: Precipath U Chargen-Nr. 701 und Precinorm UPX Chargen-Nr. 704 von Boehringer Mannheim.

3. Flüssiges Kontrollserum Fluinorm N Chargen-Nr. $1601 \mathrm{E}$ von Behringwerke AG (Marburg).

\section{Lösungen}

1. Enteiweißungsmittel (gleich für beide Methoden): $0,33 \mathrm{~mol} / 1$ Perchlorsäure.

\section{Glucosedehydrogenase-Methode}

2. Reaktionslösung: zwei Fläschchen Enzymgemisch in 11 Puffer lösen. Konzentrationen in der Reaktionslösung: 0,12 mol/1

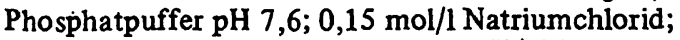
$5,2 \mathrm{kU} / 1$ Glucosedehydrogenase; $110 \mathrm{U} / 1$ Mutarotase (EC 5.1.3.3). Haltbarkeit bei Raumtemperatur mindestens zwei Monate, bei $+4{ }^{\circ} \mathrm{C}$ mindestens fünf Monate.

3. Startreagenz: eine Flasche Coenzym in $100 \mathrm{ml}$ Puffer lösen. Konzentration im Startreagenz: 5,5 mmol/1 NAD ${ }^{+}$. Haltbarkeit wie bei der Reaktionslösung.

\section{Hexokinase/Glucose-6-phosphatdehydrogenase-Methode}

4. Vorbereitung der Lösungen 1 bis 4 aus Test-Combination, nach Angaben des Herstellers lt. Begleitzettel. Haltbarkeit siehe Begleitzettel.

5. Herstellung des Reaktionsgemisches: In Puffer, der mit 100 $\mathrm{ml}$ dest. Wasser verdünnt ist, wie folgt pipettieren: $8 \mathrm{ml}$ Lösung 2 (NADP ${ }^{+}$); $8 \mathrm{ml}$ Lösung 3 (ATP); $2 \mathrm{ml}$ unverdünnte Lösung 4 (Hexokinase EC 2.7.1.1 und Glucose-6-phosphatdehydrogenase EC 1.1.1.49). Haltbarkeit des Reaktionsgemisches bei $+4^{\circ} \mathrm{C}$ mindestens 1 Tag, bei Raumtemperatur etwa 7 Stunden. Konzentration des Reaktionsgemisches: 300 mmol/1 Triethanolaminpuffer $\mathrm{pH} 7,5 ; 4 \mathrm{mmol} / 1 \mathrm{MgSO}_{4}$; $0,32 \mathrm{mmol} / 1 \mathrm{NADP}+; 0,4 \mathrm{mmol} / 1 \mathrm{ATP} ; \geqslant 1,15 \mathrm{kU} / 1$ Hexokinase; $\geqslant 1,15 \mathrm{kU} / 1$ Glucose-6-phosphatdehydrogenase.

6. Glucose-Standardlösungen in den Konzentrationen 33,3; 38,$85 ; 44,4 ; 49,95$ und $55,5 \mathrm{mmol} / 1$ sind durch Auswiegen aus wasserfreier Glucose in kalt gesättigter BenzoesäureLösung hergestellt.

7. Glucose-Standardlösungen in den übrigen Konzentrationen stammten von E. Merck (siehe Reagenzien) oder sind durch Zusammenmischen oder Verdünnen mit Benzoesäure-Lösung vorbereitet. Die Haltbarkeit aller Standardlösungen bei Raumtemperatur ist praktisch unbegrenzt.

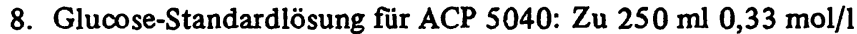
Perchlorsäure gibt man $10 \mathrm{ml} 11,1 \mathrm{mmol} / 1$ Glucoselösung. Die Glucosekonzentration der Lösung ist $426,9 \mu \mathrm{mol} / 1$. Dieser Standard wird für geräteinterne Faktor-Ermittlung unverdünnt verwendet. Im Kühlschrank aufbewahren.

\section{Materialien}

1. Glaskapillaren Blaubrand intraEND $(20 \mu l)$ kauften wir von der Fa. Brand (Wertheim).

2. Die verschließbaren Reaktionsgefäße 3810 stammten von der Fa. Eppendorf.

3. Die Lanzetten sind Erzeugnisse der Fa. Feather aus Japan.

\section{Geräte}

1. Eppendorf Gerätebau (Hamburg): Analysenautomat ACP 5040, Endpunktautomat 5030, Kettenmischer 5431, Zentrifuge 5411, Zentrifuge 3200, Reagenz-Dosierer 5210, Mikroliterpipetten (Eppendorf).

2. Diehl Datensysteme GmbH (Nürnberg): für statistische Rechnungen: Alphatronic mit Magnetbandstation und ProgrammPaket „Statistik I“.

\section{Probengewinnung}

Die Blutentnahme erfolgte mit $20 \mu l$ Glaskapillaren, die sofort in $500 \mu \mathrm{l}$ Perchlorsäure hineingebracht und gut durchgeschüttelt wurden. Im Labor wurden die Gefäße in Probenketten sortiert, nochmals mit dem Kettenmischer geschüttelt, anschließend in der Kettenzentrifuge zentrifugiert. Plasma, Serum, Vollblut, Liquor und Urin (bis zu einer Glucosekonzentration von $55,5 \mathrm{mmol} / 1$ für Glucosedehydrogenase-Methode ohne Verdünnung) können ebenfalls verwendet werden.

\section{Einstellung des ACP 5040}

Beim ACP 5040 wird die Kinetik aus vier Meßpunkten ermittelt, wobei die Meßpunkte, abhängig vom Takt des Gerätes, aus mehreren, in $80 \mathrm{~ms} \mathrm{Abstand} \mathrm{festgestellten} \mathrm{Einzelmessungen}$ gebildet worden sind. Bei Taktzeit $12 \mathrm{~s}$ bedeutet das 21 Einzelmessungen pro Meßpunkt (84 Einzelmessungen).

Der ACP 5040 ist wie folgt programmiert:

Name: Gluc; Einheit: $\mathrm{mmol} / 1 \mathrm{(mg} / \mathrm{dl})$; Temperatur: $37^{\circ} \mathrm{C}$; Takt: $12 \mathrm{~s}$; Wellenlänge: $\mathrm{Hg} 334 \mathrm{~nm}$; Abgleich E: 0,0; Standard: 11,1 mmol/1 (200 mg/dl); Rotordrehung: 1; Spülposition: 19; Startposition: 1; Volumenposition 1: $250 \mu \mathrm{l}$ (Reaktionslösung); Volumenposition 2: $50 \mu \mathrm{l}$ (enteiweißter Überstand); Volumenposition 5: $50 \mu \mathrm{l}$ (Startreagenz).

\section{Kalibrierung des ACP 5040}

Bei der kinetischen Substratbestimmung muß man mit einem Standard arbeiten. Dazu wird bei der Programmierung der Methode diar numerische Wert des Standards eingegeben. In 3-5 speziellen Kettengliedern (Farbe violett) werden die Standards (Lösung 8) vor die Serie plaziert. Die speziellen violetten Kettenglieder übertragen den Impuls = Standard an das Gerät. Das Gerät mißt bei diesen Proben die für jedes Kettenglied dazugehörenden $\Delta \mathrm{A} / \mathrm{min}$. Daraus wird ein Mittelwert gebildet, die Werte auf $\pm 5 \%$ Abweichung überprüft und aufgrund der eingespeicherten Standardkonzentration ein interner Faktor ermittelt. Damit werden dann alle weiteren Proben-Absorptionen multipliziert.

\section{Ergebnisse}

\section{Linearität, Präzision und Richtigkeit}

Die Linearität wurde mit Primärstandard von 0,555 bis $55,5 \mathrm{mmol} / 1$ überprüft. Die Messungen zeigten eine gute Linearität im ganzen Bereich. $N=15 ; y=0,1306$ $+0,9861 \mathrm{x} ; \mathrm{r}=0,9999 ; \overline{\mathrm{x}}=20,89 ; \overline{\mathrm{y}}=20,73$; $\mathrm{s}_{\mathrm{xy}}= \pm 0,239 \mathrm{mmol} / 1$.

Die Präzision der kinetischen Glucosebestimmung wurde mit verschiedenen Standardlösungen und Kontrollproben an mehreren Tagen bestimmt (Tab. 1).

Die Präzisions- und Richtigkeitskontrolle für die Hexokinase/Glucose-6-phosphatdehydrogenase-Endpunktme thode stammt aus unserer Routine und umfaßt den gleichen Vergleichszeitraum wie die kinetische Glucosedehydrogenase-Me thode (Tab. 2).

Die Nachweisgrenze ist nach Kaiser (zitiert nach 1.c. (4)) und kürzlich empfohlen von 1.c. (5), an 20 Tagen als Doppelbestimmung ermittelt worden. Dabei wurde Wasser als Probe unter gleichen Bedingungen analysiert. Umgerechnet in die benutzte Einheit ist die dreifache Standardabweichung $0,16 \mathrm{mmol} / 1(\mathrm{n}=40)^{.}$. 
Tab. 1. Präzision der kinetischen Glucosebestimmung mit ACP 5040.

Die Proben wurden in jeder Serie, wie unter Methodik beschrieben, analysiert. Bei Aliquot I und II handelt es sich um zwei Poolseren, wobei Aliquot I mit Benzoesäure 1:2 verdünnt und Aliquot II Glucose zugesetzt wurde. Die beiden Proben waren im Verhältnis 1:26 mit Perchlorsäure enteiweißt und in kleineren Portionen tiefgefroren. Täglich wurde je ein tiefgefrorenes Röhrchen Aliquot I, Aliquot II und Precipath'U aufgetaut und untersucht. Precinorm UPX wurde täglich frisch gelöst, Fluinorm $\mathrm{N}$ im Kühlschrank und die wäßrigen Standards bei Raumtemperatur aufbewahrt. Jede Probe wurde zweifach bestimmt, um eine eventuelle Verschleppung auszuschalten. In die Berechnung wurden nur die zweiten Werte einbezogen.

\begin{tabular}{|c|c|c|c|c|c|c|c|c|c|c|}
\hline \multirow{2}{*}{$\begin{array}{l}\text { Probe } \\
\text { (Sollwert in } \mathrm{mmol} / \mathrm{l} \text { ) }\end{array}$} & \multicolumn{5}{|c|}{ Präzision in der Serie } & \multicolumn{5}{|c|}{ Präzision von Tag zu Tag } \\
\hline & $\mathbf{n}$ & $\begin{array}{l}\bar{x} \\
(\mathrm{mmol} / 1)\end{array}$ & $\begin{array}{l}\mathrm{s} \\
(\mathrm{mmol} / \mathrm{l})\end{array}$ & $\begin{array}{l}\text { VK } \\
(\%)\end{array}$ & $\begin{array}{l}\mathrm{R} \\
(\%)\end{array}$ & $\mathrm{n}$ & $\begin{array}{l}\bar{x} \\
(\mathrm{mmol} / \mathrm{l})\end{array}$ & $\begin{array}{l}\mathrm{s} \\
(\mathrm{mmol} / \mathrm{l})\end{array}$ & $\begin{array}{l}\text { VK } \\
(\%)\end{array}$ & $\begin{array}{l}\mathrm{R} \\
(\%)\end{array}$ \\
\hline $\begin{array}{l}\text { Precinorm UPX } \\
\text { Fluinorm N }(6,22) \\
\text { Precipath U }(11,3) \\
\text { wäßr. Standard }(11,1) \\
\text { wäßr. Standard }(16,65) \\
\text { wäßr. Standard }(27,75) \\
\text { wäßr. Standard }(55,5) \\
\text { Aliquot I } \\
\text { Aliquot II }\end{array}$ & $\begin{array}{l}40 \\
17 \\
38 \\
40 \\
40 \\
39 \\
37 \\
18 \\
20\end{array}$ & $\begin{array}{r}5,73 \\
6,26 \\
11,27 \\
11,12 \\
16,69 \\
27,77 \\
55,05 \\
2,71 \\
52,12\end{array}$ & $\begin{array}{l}0,08 \\
0,15 \\
0,22 \\
0,16 \\
0,28 \\
0,37 \\
0,59 \\
0,08 \\
0,47\end{array}$ & $\begin{array}{l}1,4 \\
2,4 \\
1,9 \\
1,4 \\
1,7 \\
1,3 \\
1,07 \\
2,8 \\
0,9\end{array}$ & $\begin{array}{l}+0,64 \\
-0,26 \\
+0,18 \\
+0,24 \\
+0,07 \\
-0,82\end{array}$ & $\begin{array}{l}21 \\
21 \\
21 \\
21 \\
21 \\
21 \\
21 \\
21 \\
21\end{array}$ & $\begin{array}{r}5,50 \\
6,15 \\
11,07 \\
11,06 \\
16,75 \\
27,11 \\
53,79 \\
2,74 \\
51,70\end{array}$ & $\begin{array}{l}0,11 \\
0,11 \\
0,09 \\
0,18 \\
0,18 \\
0,35 \\
0,66 \\
0,09 \\
0,73\end{array}$ & $\begin{array}{l}1,9 \\
1,8 \\
0,9 \\
1,7 \\
1,1 \\
1,3 \\
1,2 \\
3,4 \\
1,4\end{array}$ & $\begin{array}{l}-1,13 \\
-2,04 \\
-0,36 \\
+0,60 \\
-2,31 \\
-3,10\end{array}$ \\
\hline
\end{tabular}

Tab. 2. Präzision und Richtigkeit der Hexokinase/Glucose-6-phosphatdehydrogenase-Endpunktmethode, die in unserer Routine verwendet wurde. Die in dieser Tabelle angeführten von Tag zu Tag-Werte stammen aus unseren Qualitätskontrollen und decken den Vergleichszeitraum. Täglich wurde mit frisch aufgelöstem Präzisionskontrollserum Precinorm UPX (ohne Sollwertangabe) und zur Richtigkeitsprüfung etwa alle 3 Tage mit flüssigem Richtigkeitskontrollserum Fluinorm N gearbeitet. Für die Prüfung der Präzision in der Serie haben wir zusätzliche Kontrollen durchgeführt. Aliquot II (s. a. Tab. 1) ist hier 1:5 verdünnt.

\begin{tabular}{|c|c|c|c|c|c|c|c|c|c|c|}
\hline \multirow{2}{*}{$\begin{array}{l}\text { Probe } \\
\text { (Sollwert in } \mathrm{mmol} / \mathrm{l} \text { ) }\end{array}$} & \multicolumn{5}{|c|}{ Präzision in der Serie } & \multicolumn{5}{|c|}{ Präzision von Tag zu Tag } \\
\hline & $\mathrm{n}$ & $\begin{array}{l}\overline{\mathbf{x}} \\
(\mathrm{mmol} / \mathrm{l})\end{array}$ & $\begin{array}{l}\mathrm{s} \\
(\mathrm{mmol} / \mathrm{l})\end{array}$ & $\begin{array}{l}\text { VK } \\
(\%)\end{array}$ & $\begin{array}{l}\mathrm{R} \\
(\%)\end{array}$ & $\mathbf{n}$ & $\begin{array}{l}\bar{x} \\
(\mathrm{mmol} / \mathrm{l})\end{array}$ & $\begin{array}{l}\mathrm{s} \\
(\mathrm{mmol} / \mathrm{l})\end{array}$ & $\begin{array}{l}\text { VK } \\
(\%)\end{array}$ & $\begin{array}{l}\mathrm{R} \\
(\%)\end{array}$ \\
\hline $\begin{array}{l}\text { Precinorm UPX } \\
\text { Fluinorm N }(6,22) \\
\text { Precipath U }(11,3) \\
\text { Aliquot II, verdünnt }\end{array}$ & $\begin{array}{l}11 \\
20 \\
18 \\
10\end{array}$ & $\begin{array}{r}5,62 \\
6,15 \\
11,28 \\
10,35\end{array}$ & $\begin{array}{l}0,08 \\
0,07 \\
0,29 \\
0,07\end{array}$ & $\begin{array}{l}1,4 \\
1,1 \\
2,6 \\
0,7\end{array}$ & $\begin{array}{l}-1,1 \\
-0,2\end{array}$ & $\begin{array}{l}31 \\
11\end{array}$ & $\begin{array}{l}5,61 \\
6,26\end{array}$ & $\begin{array}{l}0,13 \\
0,08\end{array}$ & $\begin{array}{l}2,3 \\
1,3\end{array}$ & $+0,6$ \\
\hline
\end{tabular}

Um einen Verschleppungsfehler festzustellen, haben wir 20 Tage nach jedem Standard $(11,1 ; 16,65 ; 27,75 ; 44,4$ und $55,5 \mathrm{mmol} / \mathrm{l}$ ) Wasser als Probe analysiert. Die ermittelte dreifache Standardabweichung bis zu Standard $27,75 \mathrm{mmol} / \mathrm{l}$ betrug umgerechnet in die verwendete Einheit $0,15 \mathrm{mmol} / 1(\mathrm{n}=20) ; \mathrm{d}$. h. sie ist gleich groß wie die untere Nachweisbarkeitsgrenze. Bei den Konzentrationen ab 33,3 und $44,4 \mathrm{mmol} / 1$ sind diese Werte 0,20 und $0,20 \mathrm{mmol} / 1, \mathrm{ab} 55,5 \mathrm{mmol} / 10,31 \mathrm{mmol} / \mathrm{l}$; somit kleiner als $1,8 \mathrm{mmol} / \mathrm{l}$, die durchschnittliche dreifache Standardabweichung für diesen Bereich (s. a. Tab. 1).

Der prozentuale Verschleppungskoeffizient (Q) wurde nach Hjelm (zitiert in 1.c. (6)) an 16 Tagen aus einer Serie von Glucosestandards 5 mal 5,$55 ; 55,5 ; 5,55$ mmol/1 bestimmt. Der prozentuale Koeffizient $Q_{1}$ (für die Verschleppung von der niedrigen zur hohen Konzentration) ist $0,36 \%$ und $\mathrm{Q}_{2}$ (für die Verschleppung von der hohen zur niedrigen Konzentration) $0,34 \%$.

Wir schließen daraus auf einen praktisch unbedeutsamen Verschleppungsfehler.

Die Wiederfindung von Glucose im Serum zeigt Tabelle 3. Die Wiederfindungsrate ist aufgrund der Ergebnisse als gut $\mathrm{zu}$ bezeichnen.
Tab. 3. Wiederfindung von Glucose im Serum. Angegeben sind die Mittelwerte aus je 5 Einzelbestimmungen. Die Aufstockungen sind anhand von zwei Patientenseren hergestellt.

\begin{tabular}{lcclcc}
\hline $\begin{array}{l}\text { Glucosekonzentration } \\
\text { berechnet } \\
\text { (mmol/l) }\end{array}$ & $\begin{array}{l}\text { gefunden } \\
\text { (mmol/1) }\end{array}$ & n & $\begin{array}{l}\text { s } \\
(\mathrm{mmol} / \mathrm{l})\end{array}$ & $\begin{array}{l}\text { VK } \\
(\%)\end{array}$ & $\begin{array}{l}\text { Prozentuale } \\
\text { Wiederfindung }\end{array}$ \\
\hline 1,47 & 1,44 & 5 & 0,045 & 3,1 & 97,9 \\
2,942 & 2,953 & 5 & 0,025 & 0,8 & 100,4 \\
5,883 & 5,905 & 5 & 0,063 & 1,1 & 100,4 \\
8,664 & 8,902 & 5 & 0,128 & 1,4 & 102,7 \\
14,774 & 14,90 & 5 & 0,290 & 1,9 & 100,9 \\
28,111 & 28,993 & 5 & 0,276 & 0,9 & 103,1 \\
35,52 & 37,263 & 5 & 0,253 & 0,7 & 104,9 \\
41,447 & 42,824 & 5 & 0,489 & 1,1 & 103,3 \\
47,558 & 47,475 & 5 & 0,210 & 0,4 & 99,8 \\
\hline
\end{tabular}

\section{Korrelation mit der Vergleich sme thode}

Die Abbildung 1 zeigt die aus 355 Wertepaaren ermittelte Korrelation mit der Hexokinase/Glucose-6-phosphatdehydrogenase-Endpunk tmethode. Die Ergebnisse sprechen für eine gute Ubereinstimmung zwischen beiden Methoden. 


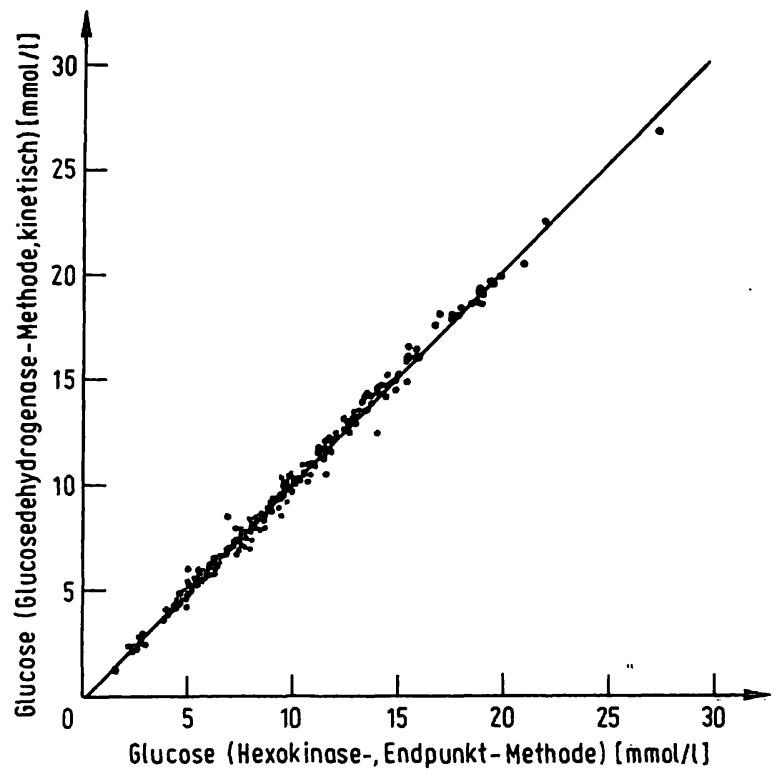

Abb. 1. Korrelation der Meßergebnisse: GlucosedehydrogenaseMethode, kinetisch (y) mit Hexokinase/Glucose-6phosphatdehydrogenase-Methode, Endpunkt (x). Der berechnete Korrelationskoeffizient aus 355 Wertepaaren lautet $\mathbf{r}=0,9967$; die Regressionsgleichung ist $y=-0,07+1,012 x ; s_{x y}= \pm 0,329 \mathrm{mmol} / 1$.

\section{Diskussion}

Die Bestimmung der Glucosekonzentration mit der Hexokinase/Glucose-6-phosphatdehydrogenase-End-

\section{Literatur}

1. Brümmer, W. \& Ebeling, W. (1976), Eigenschaften und Anwendung der Glucose-Dehydrogenase. Kontakte 2, 3-7, E. Merck AG, Darmstadt.

2. Ziegenhorn, J. (1977), In: Grundlagen der enzymatischen Analyse (Bergmeyer, H. U., ed.), 1. Aufl., pp. 81-83, Verlag Chemie, Weinheim, New York.

3. Müller-Matthesius, R. (1975), Z. Klin. Chem. Klin. Biochem. 13, 169-170. punktmethode wird gewöhnlich aus eiweißfreiem Uberstand, der nach der Behandlung der Proben mit Perchlorsäure entsteht, durchgeführt. Um Pipettierungs- und Kapillarfüllungsfehler bei unseren Vergleichsuntersuchungen ausschalten zu können, enteiweißten wir nicht wie für die Glucosedehydrogenase-Methode vorgeschrieben mit Perchlorsäure-Perchloratlösung, sondern mit Perchlorsäure.

Dabei fanden wir, daß die theoretisch notwendige $\mathrm{NAD}^{+}$-Konzentration nur bis zu einer Glucosekonzentration von etwa $44 \mathrm{mmol} / \mathrm{l}$ ausreicht. Um eine Linearität bís $55,5 \mathrm{mmol} / 1$ Glụcose erreichen zu können, mußten wir die $\mathrm{NAD}^{+}$-Konzentration auf $5,5 \mathrm{mmol} / 1$ erhöhen.

Die Ergebnisse zeigen, daß ein niedriger $\mathrm{pH}-$ Wert des Überstandes unter den gewählten Kriterien nicht stört.

Unter den beschriebenen Bedingungen sind 300 Proben in einer Stunde zuverlässig bestimmbar.

\section{Danksagung}

Der Fa. E. Merck danken wir für das Überlassen von Reagenzien.

4. Haeckel, R. \& Haeckel, H. (1972), Z. Klin. Chem. Klin. Biochem. 10, 453-461.

5. Stamm, D. (1979), Recommendations for the Description of a Selected Method in Clinical Chemistry. J. Clin. Chem. Clin. Biochem. 17, 180-282.

6. Haeckel, R. (1975), In: Qualitätssicherung im medizinischen Labor (Haeckel, R., ed.), 1. Aufl., pp. 133-135, Deutscher Ärzte-Verlag GmbH, Köln-Lövenich.

Dipl. Ing. med. Biochem. Aladár Bruckner Städtisches Krankenhaus - Zentrallabor Gotenstraße 6-8

D-6230 Frankfurt/M.-Höchst 